\title{
SPECTRUM OF FUNGAL ISOLATES IN DIFFERENT CLINICAL SAMPLES
}

\author{
Dibya Prasana Mohanty1, Susanta Kumar Sahu², Uday Hembram³ \\ ${ }^{1}$ Assistant Professor, Department of Microbiology, SCB Medical College, Cuttack. \\ ${ }^{2}$ Professor, Department of Microbiology, SLN Medical College, Koraput, Odisha. \\ ${ }^{3}$ Tutor, Department of Microbiology, VSS Institute of Medical Sciences and Research, Burla.
}

\begin{abstract}
\section{BACKGROUND}

Fungal infections are on the rise due to changing practices in medical care; increase in the immunocompromised states, and due to increase in the use of antibiotics and steroid therapy. Currently, the awareness levels among the medical personnel regarding fungal diseases are lacking. More and more cases of fungal infections are being reported from cases of AIDS, malignancy, and transplant recipients, patients in ICU or on steroid therapy. Indiscriminate use of antibiotic therapy is another reason for the growing incidence of fungal infections.
\end{abstract}

\section{MATERIALS AND METHODS}

A retrospective study was undertaken to know the burden of fungal infection in different clinical samples. A total number of 3146 clinical samples from different in-patient departments processed in the microbiology laboratory of a tertiary care teaching hospital were analysed. The samples were processed for microscopy by KOH mount, Gram stain, negative stain, Lactophenol cotton blue stain and culture on SDA with Chloramphenicol, BHI broth (for blood), Dalmau culture on CMA, Slide culture and relevant biochemical tests depending on the type of sample and were identified by standard algorithm.

\section{RESULTS}

It was seen that most of the isolates belonged to yeasts comprising C. albicans (45\%) followed by C. tropicalis (33.6\%). The samples from the genital tract, oropharyngeal swabs, blood, urine, CSF and faeces showed no isolation of filamentous fungi. Moulds were mainly isolated from respiratory samples, skin and its appendages, aural swabs and deep wounds. Dermatophytes formed the major isolates from skin and its appendages.

\section{CONCLUSION}

A more detailed study is needed to know the socio-epidemiological prevalence of these infections and their burden in the community and in hospitalised patients.

\section{KEYWORDS}

Fungi, Yeast, Filamentous Fungi, Candida.

HOW TO CITE THIS ARTICLE: Mohanty DP, Sahu SK, Hembram U. Spectrum of fungal isolates in different clinical samples. J. Evolution Med. Dent. Sci. 2017;6(69):4906-4911, DOI: 10.14260/Jemds/2017/1064

\section{BACKGROUND \\ The fungi belong to a group of microorganisms with characteristics different from other disease-causing organisms like bacteria and parasites. Among the 1.5 million fungal species described, only about 300 species are associated with human disease and only about a 100 of them are capable of causing infection in normal individuals.1, 2 With changing practices in medical care, fungal infections have been on the rise. Availability of improved diagnostic methods also helps us to conclusively identify fungal infections, which were earlier not possible, and were attributed to other organisms. Fungi are implicated in various allergy, infectious diseases and toxic manifestations. The pattern of fungal diseases varies according to various geophysical and climatic condition of a particular area.}

Financial or Other, Competing Interest: None.

Submission 24-07-2017, Peer Review 18-08-2017,

Acceptance 23-08-2017, Published 28-08-2017.

Corresponding Author:

Dr. Susanta Kumar Sahu,

Professor, Department of Microbiology,

SLN Medical College,

Koraput-764020, Odisha.

E-mail: drsusantavss@gmail.com

DOI: $10.14260 /$ jemds $/ 2017 / 1064$
Although superficial fungal infections are very common in human population they are usually easy to diagnose, and are curable. It is the rather uncommon invasive infections which are not only life threatening, but also difficult to diagnose. ${ }^{3}$

Fungal infections of human beings originate mostly from exogenous sources and are acquired mainly by inhalation and traumatic implantation. Some infections particularly of Candida are mostly derived from endogenous sources. Last few decades have witnessed an alarming rise in the incidence of fungal infections particularly in cases of AIDS, malignancy, transplant recipients, patients in ICU, and patients on steroid therapy. Indiscriminate use of antibiotics has led to the suppression of the local microflora, which has helped in the growing incidence of fungal infections. ${ }^{4,5,6}$ Besides this, fungi pose a difficult situation from the clinicians' point of view because of non-availability of proper laboratory facilities, limited number of antifungals available, their side effects and the long duration of treatment needed to eradicate the infection. As most of these fungi are ubiquitous and freeliving in the environment it is very difficult on the part of the laboratory to prove the cause effect relationship between the fungus and the disease they are attributed to. Although fungal infections contribute substantially to human morbidity and mortality, their burden on human health is not widely scientifically studied. We also lack in providing efficient 
diagnostic facilities in most clinical laboratories and lag behind in research in this field.

Keeping this in view a retrospective analysis of data regarding the distribution of different types of fungi in various clinical samples processed in the mycology laboratory was undertaken in a tertiary care teaching hospital in Odisha.

\section{MATERIALS AND METHODS}

\section{Type of Study}

Retrospective, Observational.

Clinical specimens processed in the Microbiology Department for mycological tests during the period from January 2015 to June 2016 were analysed. No record or history of taking antifungal treatment by any patient was observed. The samples included respiratory tract samples, blood, urine, corneal scrapings, specimens from skin and its appendages (Nail and Hair), oropharyngeal swabs, faeces, aural swabs, pus \& wound swabs, high vaginal swabs, cerebrospinal fluid (CSF) etc. Respiratory samples included sputum, bronchoalveolar lavage fluid (BAL), pleural fluid and endotracheal aspirates.

Samples were examined for microscopy and culture. Microscopic examination included $10 \% \mathrm{KOH}$ mount, Gram stain, wet mount \& Negative staining by Nigrosin depending upon the type of sample. The blood samples were cultured in Brain Heart Infusion (BHI) broth and incubated for 4 weeks. Subcultures from blood were made periodically onto Sabouraud Dextrose Agar (SDA) and incubated at room temperature for 2 weeks before they were discarded as negative. Centrifuged CSF deposits were cultured in SDA. The other samples were inoculated in two sets of SDA with chloramphenicol, one set incubated at $22-25^{\circ} \mathrm{C}$ and the other set at $37^{\circ} \mathrm{C}$ for 6 weeks before discarding them as negative.

Yeast like colonies isolated were stained with Gram stain to know the shape, budding, presence of pseudohyphae, capsule etc. Yeast like colonies from CSF samples \& wound swabs and respiratory samples were also examined by negative staining to rule out Cryptococcus. The other yeast colonies were subjected to a panel of tests, namely morphological tests, by Germ tube test, Cut streak (Dalmau) culture, sugar fermentation, sugar assimilation test, urease test, surface growth etc. Standard algorithm was used for identification of these organisms. Dalmau culture plate was done by inoculating the yeast on Corn meal agar (CMA) by cut streak, and incubating the plate at $20^{\circ} \mathrm{C}$ for 4 weeks after covering the inoculated area by a sterile glass coverslip. This helps to see the ability of the yeasts to produce mycelium or pseudo-mycelium and enhances Chlamydospore production in C. albicans. It also helps production of Arthrospores by Trichosporon. The plate is observed periodically under an inverted microscope.7,8 Rhodotorula was identified by its typical salmon pink coloured colonies and absence of mycelium in Dalmau preparation.

Filamentous fungi were identified by gross morphology of the colony on the SDA medium and microscopic appearance of the teased-out growth in Lactophenol cotton blue mount and by slide culture method. 7,8

After the processing was over the clinical specimens and culture were discarded in discard jars containing chemical disinfectants (Cresol 5\% usually); these were later on taken by personnel from hospital biomedical waste management department for further treatment.

\section{RESULTS}

The results of the study are depicted in Table 1 . We analysed 3146 clinical specimens collected during the period from January 2015 to June 2016. The overall proportion of fungal isolates is $15.1 \%$ comprising predominantly (12.9\%) of yeasts, while filamentous fungi contributed a minor portion (2.2\%). It was observed that maximum percentage of fungal isolates was obtained from aural swabs (46.4\%), vaginal discharges $(35.4 \%)$, corneal scrapings $(32.1 \%)$, respiratory samples $(28.5 \%)$, skin and its appendages $(27.1 \%)$ and faeces samples (23.9\%). Urine and blood which constituted more than half the samples showed isolation rates of $13.5 \%$ and $13.3 \%$ respectively. All of these isolates belonged to yeasts only. Least proportion of fungal isolation was seen in wound swabs \& pus, CSF, and oropharyngeal swabs where the respective isolation rates were $5 \%, 9.1 \%$ and $10.6 \%$ respectively.

Filamentous fungi were the major isolates from corneal scrapings, aural swabs and skin scrapings with a proportion of $25 \%, 25 \%$ and $19.6 \%$ respectively, in comparison to yeast isolates where the comparative values were $7.1 \%, 21.4 \%$ and $7.5 \%$. Yeasts were the only isolates from urine, blood vaginal discharge, oropharyngeal swabs and faeces, and formed a major portion from respiratory samples, wound swabs \& pus. CSF showed isolation of 2 numbers of Cryptococcus neoformans from 22 samples.

The distribution of different yeasts and moulds isolated from the clinical samples are summarised in the Tables $2 \& 3$. Candida is the major isolate among the yeasts, with a minority contributed by others like Trichosporon, Geotrichum, Rhodotorula and Blastoschizomyces spp. Among the Candida spp., C. albicans contributed to $45 \%$, whereas non-albicans Candida (NAC) were isolated in the remaining $55 \%$. Most of the NAC belonged to C. tropicalis, C. parapsilosis and C. krusei. Among the filamentous fungi isolated, Aspergillus is the major isolate. Most of the isolates from corneal scrapings (25\%) belonged to environmental moulds, namely Fusarium (2), Aspergillus (2), Penicillium (1), Curvularia (1). A rare isolate Blastoschizomyces (1) was also isolated from corneal scrapings.

Dermatophytes, namely members of the genus Trichophyton, Epidermophyton and Microsporum were the major isolates from skin and its appendages as shown in Table 4 , whereas only 8 out of the total 29 isolates from skin and its appendages were Candida spp. [Candida albicans (3) and NAC (5)].

\begin{tabular}{|c|c|c|c|c|}
\hline \multirow{2}{*}{$\begin{array}{c}\text { Clinical } \\
\text { Sample }\end{array}$} & \multirow{2}{*}{$\begin{array}{c}\text { No. of } \\
\text { Samples }\end{array}$} & Yeasts & $\begin{array}{c}\text { Mycelial } \\
\text { Fungi }\end{array}$ & $\begin{array}{c}\text { Total } \\
\%\end{array}$ \\
\hline Urine & 1224 & $163(13.3)$ & 0 & 13.3 \\
\hline $\begin{array}{c}\text { Wound swabs \& } \\
\text { Pus }\end{array}$ & 685 & $28(4.1)$ & $6(0.9)$ & 5.0. \\
\hline Blood & 453 & $61(13.5)$ & 0 & 13.5 \\
\hline $\begin{array}{c}\text { Respiratory } \\
\text { samples }\end{array}$ & 375 & $86(22.9)$ & $21(5.6)$ & 28.5 \\
\hline Skin scrapings & 107 & $8(7.5)$ & $21(19.6) *$ & 27.1 \\
\hline $\begin{array}{c}\text { Oropharyngeal } \\
\text { scrapings }\end{array}$ & 85 & $9(10.6)$ & 0 & 10.6 \\
\hline Vaginal Discharge & 65 & $23(35.4)$ & 0 & 35.4 \\
\hline
\end{tabular}




\begin{tabular}{|c|c|c|c|c|}
\hline Aural swabs & 56 & $12(21.4)$ & $14(25)$ & 46.4 \\
\hline Faeces & 46 & $11(23.9)$ & 0 & 23.9 \\
\hline Corneal scrapings & 28 & $2(7.1)$ & $7(25)$ & 32.1 \\
\hline CSF & 22 & $2(9.1) \dagger$ & 0 & 9.1 \\
\hline Total & 3146 & $405(12.9)$ & $69(2.2)$ & 15.1 \\
\hline Table 1. Distribution of Yeast and Mycelial Fungi among \\
Different Clinical Samples \\
\hline
\end{tabular}

† All the 2 isolates are Cryptococcus neoformans

\begin{tabular}{|c|c|}
\hline C. albicans & $182(45.0)$ \\
\hline C. tropicalis & $136(33.6)$ \\
\hline C. parapsilosis & $47(11.6)$ \\
\hline C. krusei & $20(4.9)$ \\
\hline C. glabrata & $4(1.0)$ \\
\hline other Candida spp. & $2(0.5)$ \\
\hline Trichosporon & $5(1.2)$ \\
\hline Geotrichum & $3(0.7)$ \\
\hline Rhodotorula & $2(0.5)$ \\
\hline B. capitatus & $1(0.2)$ \\
\hline Cryptococcus & $3(0.7)$ \\
\hline Total & $\mathbf{4 0 5}$ \\
\hline Table 2. Distribution of the Yeasts \\
\hline
\end{tabular}

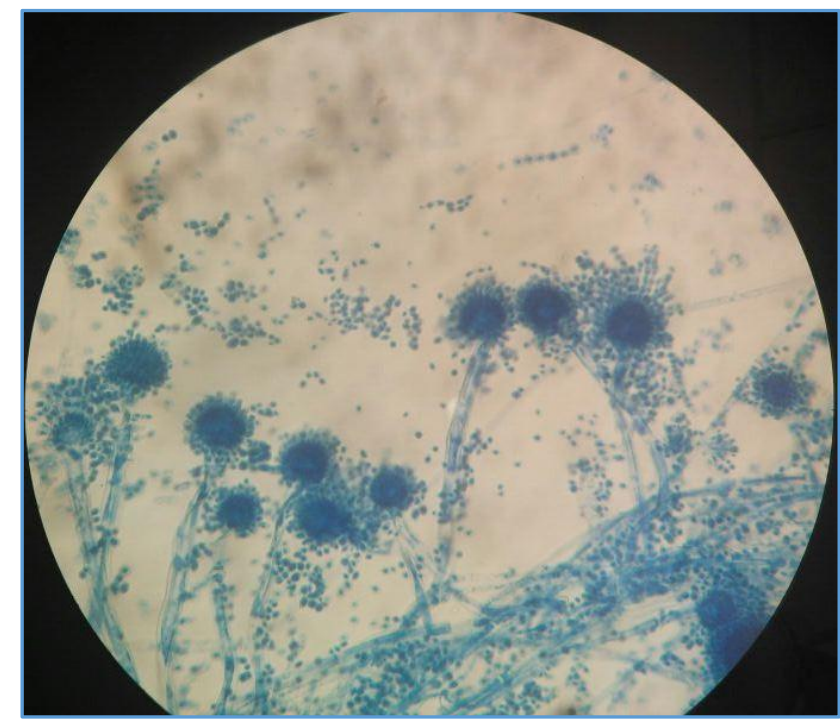

A. flavus-LPCB mount

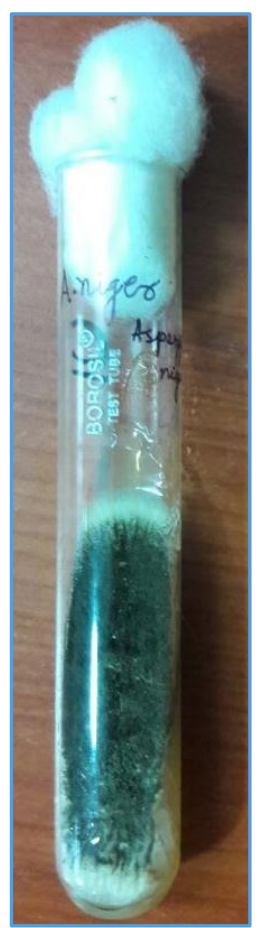

A. niger-on SDA

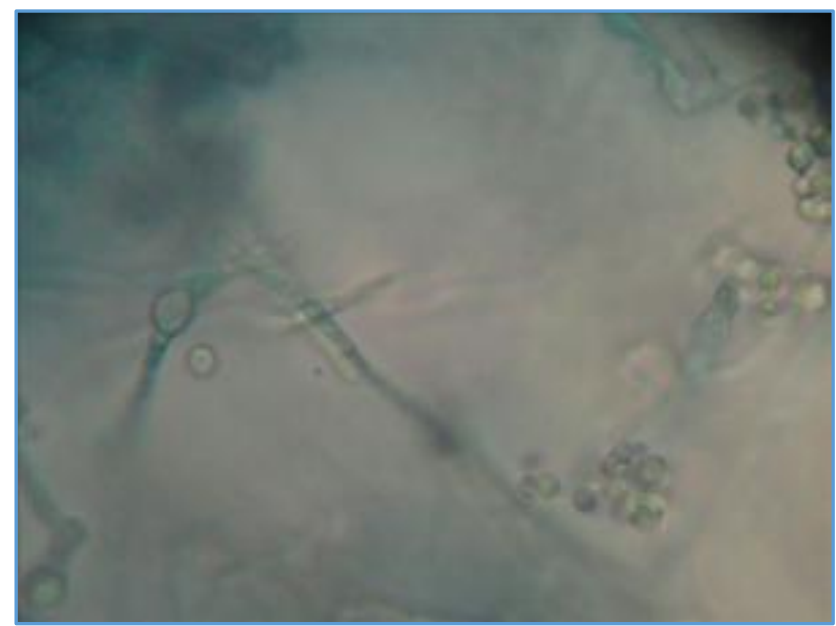

C. albicans-Chlamydospore- in Dalmau Preparation 


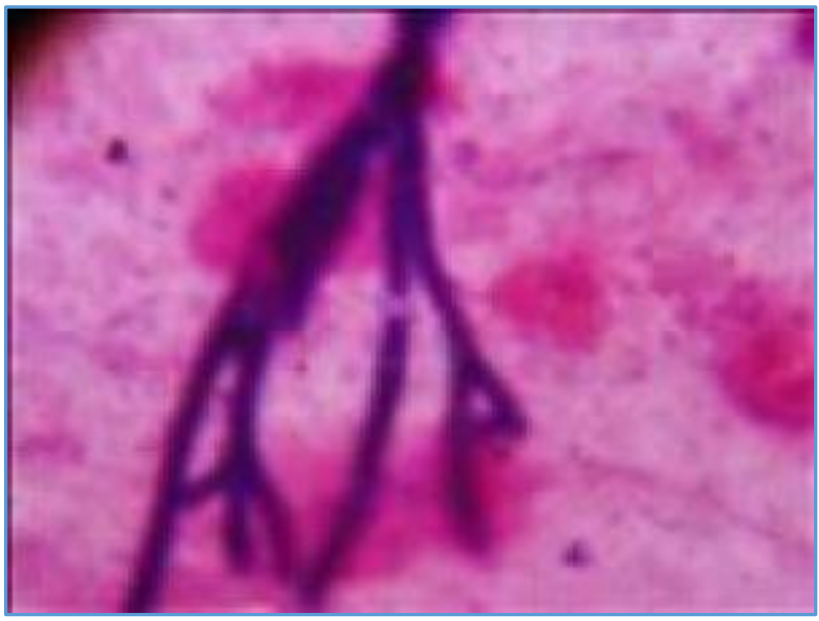

Candida -Pseudohyphae-Gram stain

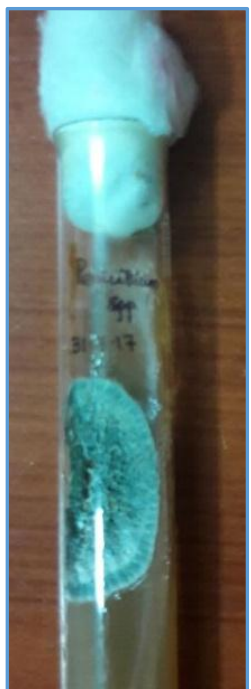

Penicillium spp.

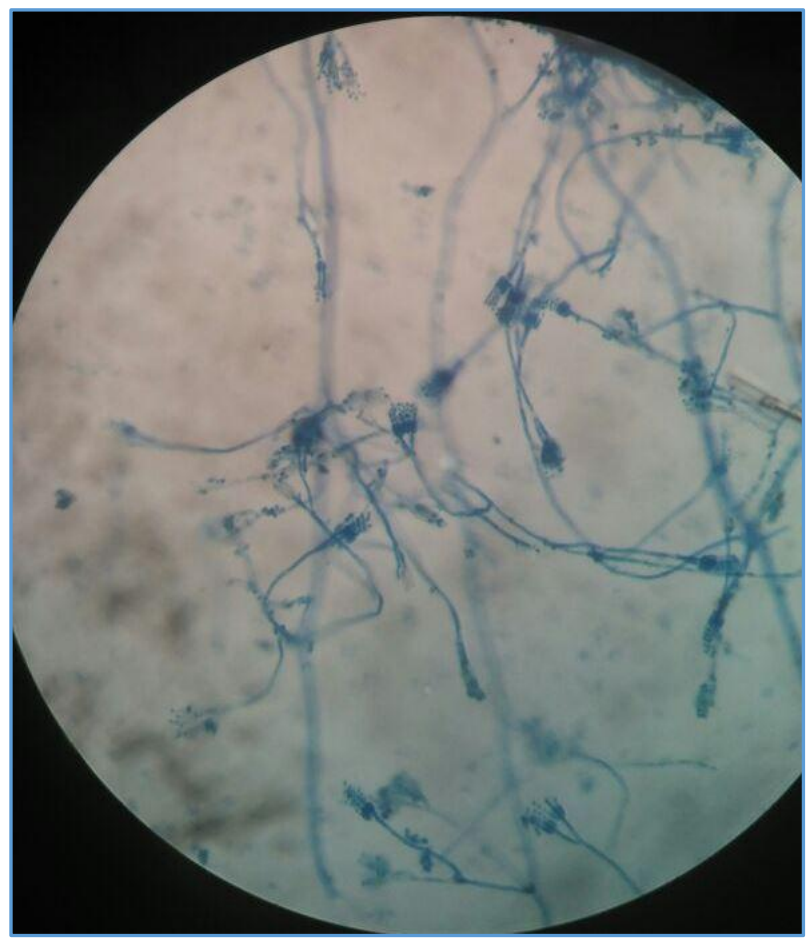

Penicillium -LPCB Mount

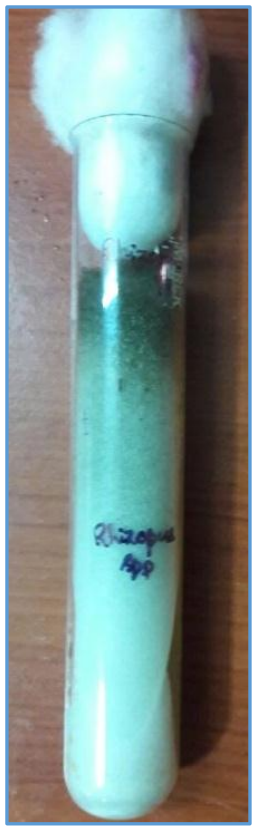

Rhizopus -on SDA

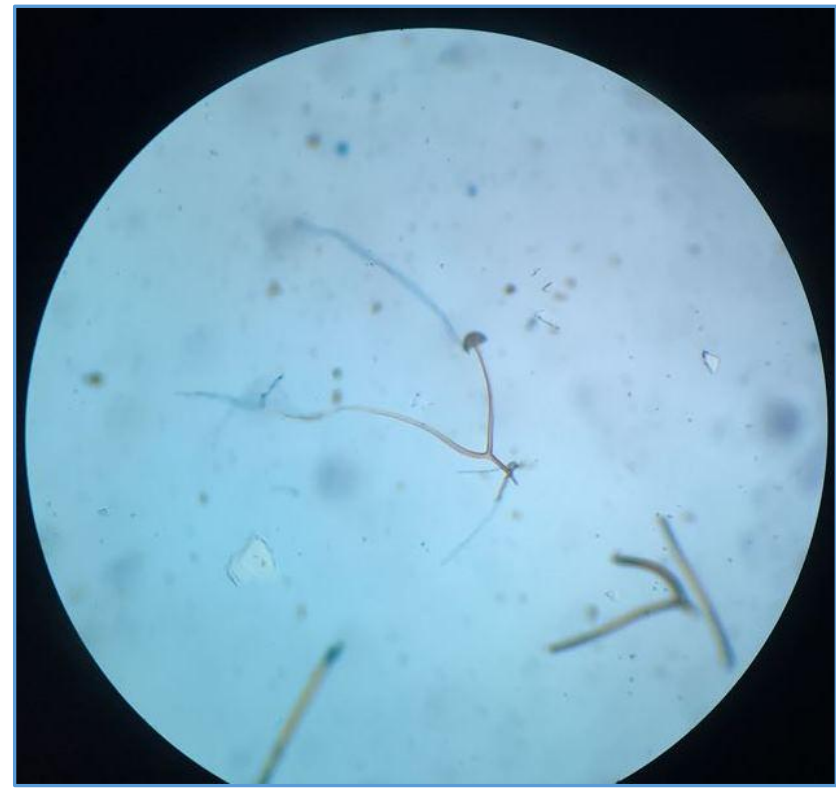

Rhizopus-LPCB mount

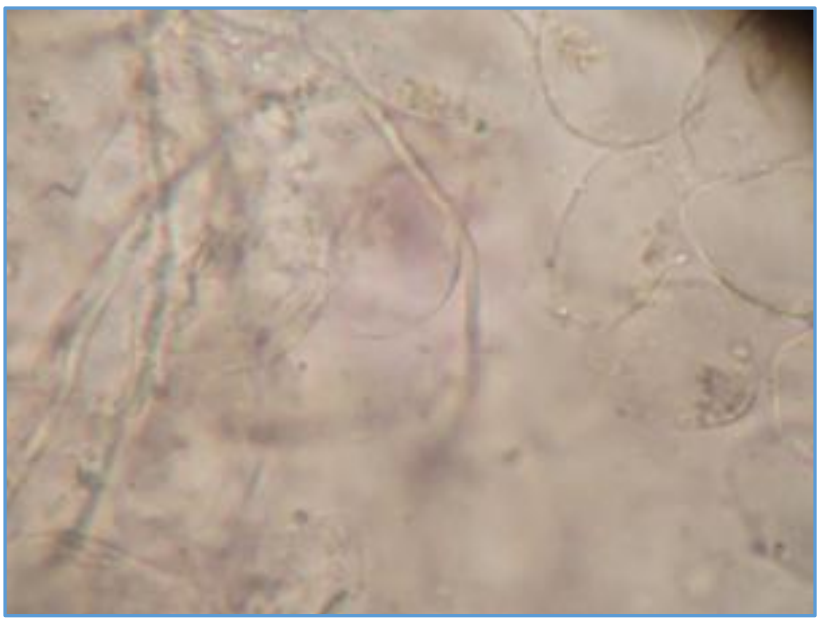

Skin Scrapings-KOH mount 


\section{DISCUSSION}

With increasing incidence of immunocompromised conditions due to AIDS, diabetes, malignancy, invasive medical procedures, transplantation, extended ICU stay, and increased life expectancy rates, the agents of mycotic infections yet to be known, and mostly environmental contaminants, have emerged as one of the important causes of infection. The use of indiscriminate use of antibiotics and steroids has further increased the diseases caused by fungi.

In the present study, we noted that the incidence of fungal isolates from clinical specimens was $15.1 \%$, consisting predominantly of yeasts (12.9\%), and in part, by filamentous fungi $(2.2 \%)$, mainly Aspergillus. Similar finding is also described by other studies. ${ }^{3,4}$

From the genital tract, oropharynx and faeces samples, Candida is the sole isolate as it is a member of the microbial flora of these regions. In these areas also, C. albicans is the major isolate followed by C. tropicalis. Similar observations were also made by Kaur et al. ${ }^{4}$

In our study, it was seen that C. albicans was the most common isolate from total samples followed by $\mathrm{C}$ tropicalis among the yeasts which is supported by the findings of others. $2,4,5$ Globally, the incidence of C. albicans is in a declining trend and incidence of the non-albicans Candida are on the rise. ${ }^{6}$ In our study also we found that the NAC comprised 209 number of Candida whereas number of $\mathrm{C}$. albicans was only 183.

Nosocomial candidaemia is one of the common morbid conditions in ICU and in neonates, it is more fatal. C. albicans is the most common cause of nosocomial blood stream infections worldwide. This is also evident from our study. In our study, it is seen that yeasts contributed $13.5 \%$ cases of blood stream infection. This might be due to the fact that the blood cultures tested in our department were mostly obtained from neonatal ICU cases, who were immunologically immature. ${ }^{9-11}$

Among the urine cultures, $13.3 \%$ showed growth of Yeasts. C. albicans is the most common isolate among them followed by C. tropicalis, C. parapsilosis and C. krusei which agrees with the findings of other researchers. ${ }^{10,12}$

In respiratory samples which comprised of sputum, BAL fluid, endotracheal aspirate and pleural fluid, the major isolate was yeasts belonging mostly to Candida spp. But a significant proportion are also contributed by filamentous fungi like Aspergillus, Penicillium and Rhizopus spp. It was observed that all the isolates from endotracheal aspirates are Candida. This finding corroborates with the observation of Kaur R et al and Dorko E et al.4,13

The pus samples collected from superficial wounds, like burn wounds and bed sores, showed mainly the growth of Candida, whereas the sample collected from deep wounds or sinuses mostly showed filamentous fungi belonging to the dematiaceous group. These results are in agreement with the findings of Dowd SE et al.13 Samples from unopened abscess cavities and surgical sites showed no growth of fungi. ${ }^{14}$

From skin and its appendages dermatophytes were the major isolates consisting of Trichophyton rubrum, $\mathrm{T}$. mentagrophyte, Microsporum and Epidermophyton spp. followed by the Candida. For obvious reasons of keratinophilic nature of the dermatophyte they predominate in keratinised tissues. ${ }^{15}$ Corneal scrapings showed filamentous fungi (25\%) belonging to Aspergillus, Penicillium, Fusarium spp. as major isolates, as also described by Thomas PA. Yeasts were only isolated in $7 \%$ of the corneal specimens. ${ }^{16}$

This study is only a preliminary observation to know the burden of the fungal infections in the hospital. More detailed multicentric prospective studies taking into account the demographic data, risk factors like immune status, history of endocrinopathy, malignancy, steroid and antibiotic therapy, occupation and socioeconomic condition of the patient should be undertaken to have a first-hand data of these diseases. These studies supported by more detailed laboratory investigations including serological tests and histopathological studies, molecular methods and antifungal sensitivity testing are needed for a clearer picture of the burden of these infections and to plan out remedial measures.

\section{CONCLUSION}

Though fungi were associated with both allergic and infectious diseases, it can be concluded from our study that a significant proportion of infections are contributed by fungus. Candida spp., particularly C. albicans was still the predominant fungal isolate in these cases in almost all samples except skin and its appendages and corneal scrapings where dermatophytic moulds and Aspergillus spp. were predominant.

Although the incidence of fungal infections is increasing with advances in the patient management techniques, the awareness among the medical fraternity in general regarding fungal pathogens is lagging behind. To counter this there is a need to carry out surveillance programmes to have an idea of the socio-epidemiological behaviour and the actual burden of these infections. The clinicians should consider including fungal pathogens in the differential diagnosis of many infectious and allergic diseases. Availability of improved diagnostic methods widely will make this more feasible and logical. More research to identify newer and safer antifungal agents and to develop vaccines against these also will go a long way in our fight against microbial infections.

\section{REFERENCES}

[1] Richardson MD, Wamock DW. Fungal infections: diagnosis and management. $3^{\text {rd }}$ edn. Massachusetts: Blackwell Publishing Ltd., 2003: p. 4.

[2] Types of Fungal diseases. Atlanta: Center for Disease Control and Prevention. 2017. https://www.cdc.gov/fungal/diseases/index.html\#on e.

[3] Weinberger M, Sacks T, Sulkes J, et al. Increasing fungal isolation from clinical specimens: experience in a university hospital over a decade. J Hosp infect 1997;35(3):185-95.

[4] Kaur R, Dhakad MS, Goyal R, et al. Spectrum of opportunistic fungal infection in HIV/AIDS patients in tertiary care hospital in India. Can J Infect Dis Med Microbiol Article ID 2373424, 2016;2016:1-7.

[5] Brown GD, Denning DW, Gow NAR, et al. Hidden killers: human fungal infections. Sci Transl Med 2012:4(165):165rv (http://texasgrassfedbeef.com/sites/default/files/pdf /human_fungal_infections_killers_by_brown_scitransl med_2012.pdf) 
[6] Guinea J. Global trends in the distribution of Candida species causing candidemia. Clin Microbiol Infect 2014:20(Suppl 6):5-10.

[7] Milne LJR. Fungi. In: Collee JG, Frase AG, Marmion BP, eds. Mackie \& McCartney practical medical microbiology. 14th edn. Edinburgh: Churchill Livingstone 1996:695-717.

[8] Fothergill AW. Medically significant fungi. In: Mahon CR, Lehman DC, Manuselis G. eds. Text book of diagnostic microbiology. $5^{\text {th }}$ edn. Missouri: Saunders/Elsevier Publishers 2015:589-622.

[9] Wisplinghoff $\mathrm{H}$, Bischoff $\mathrm{T}$, Tallent $\mathrm{SM}$, et al. Nosocomial bloodstream infections in US hospitals: analysis of 24, 179 cases from a prospective nationwide surveillance study. Clin Infect Dis 2004;39(3):309-17.

[10] Pahwa N, Kumar R, Nirkhiwale S, et al. Species distribution and drug susceptibility of Candida in clinical isolates from a tertiary care centre at Indore. Indian J Med Microbiol 2014;32(1):44-8.
[11] Giri S, Kindo AJ. A review of Candida species causing blood stream infection. Indian J Med Microbiol 2012;30(3):270-8.

[12] Sukumaran J, Sundaram JM, Sivan RR. Changing trend in the clinical distribution of Candida species in a tertiary care hospital. J NTR Univ Health Sci 2012;1(4):222-6.

[13] Dorko E, Pilipcinec E, Tkasikova L. Fungal diseases of the respiratory tract. Folia Microbiol 2002;47(3):3024.

[14] Dowd SE, Hanson JD, Rees E, et al. Survey of fungi and yeast in polymicrobial infections in chronic wounds. J Wound Care 2010;20(1):40-7.

[15] Sharma M, Sharma R. Profile of Dermatophytic and other fungal infections in Jaipur. Ind J Microbiol 2012;52(2):270-4.

[16] Thomas PA. Fungal infections of the cornea. Eye (Lond) 2003;17(8):852-62. 